\title{
Para-(benzoyl)-phenylalanine as a potential inhibitor against leptospirosis
}

\author{
Dibyabhaba Pradhan*, Vani Priyadarshini, Manne Munikumar and Amineni Umamaheswari** \\ SVIMS Bioinformatics Centre (BIF), SVIMS University, Tirupati-517507. \\ *Presenting Author; **Corresponding Author; Email: svims.btisnet@nic.in.
}

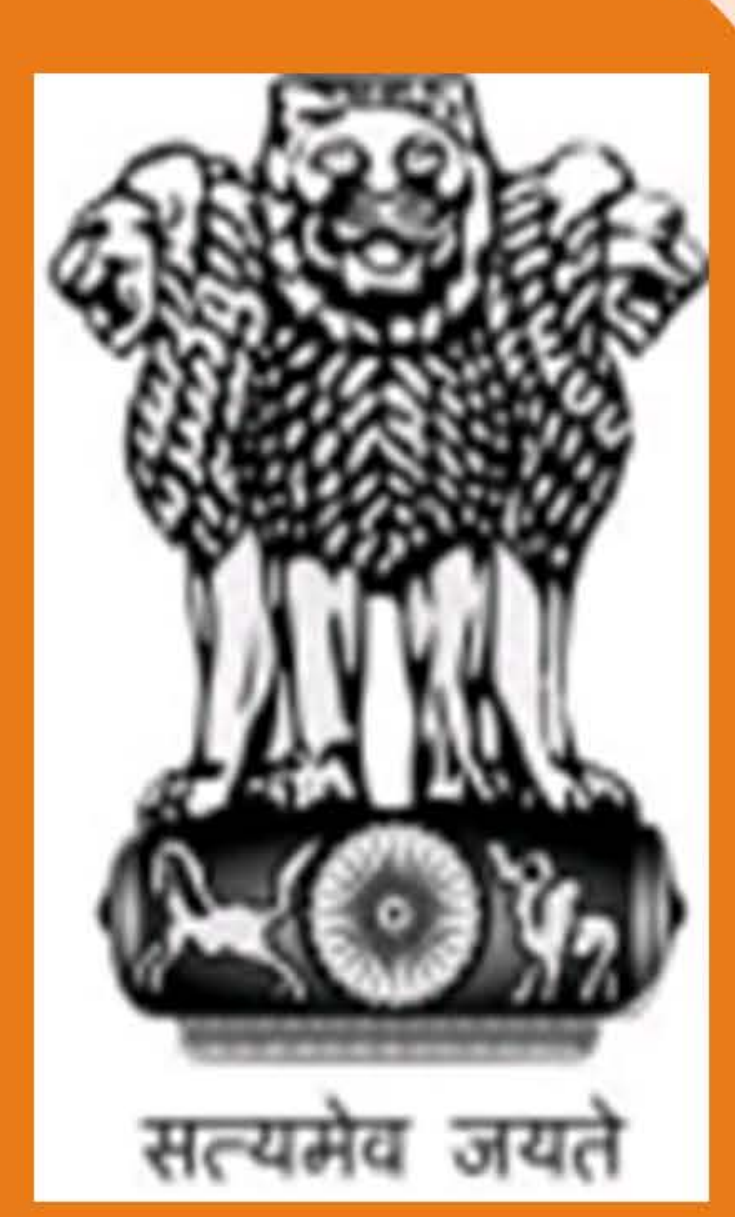

\section{Key points}

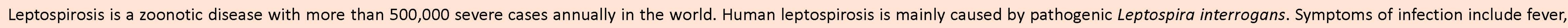

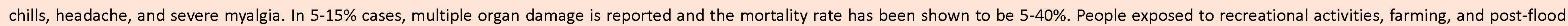

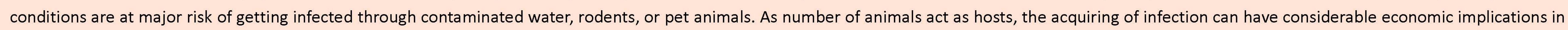
developing countries like India .

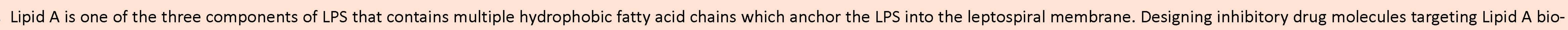
synthesis would dissolve the structural integrity of membrane structure leading to cell lysis and death of Leptospira.

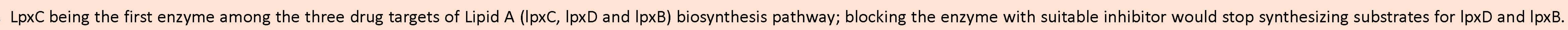

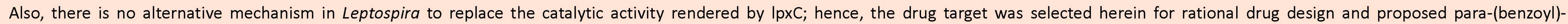
phenylalanine as a potential inhibitor against leptospirosis.

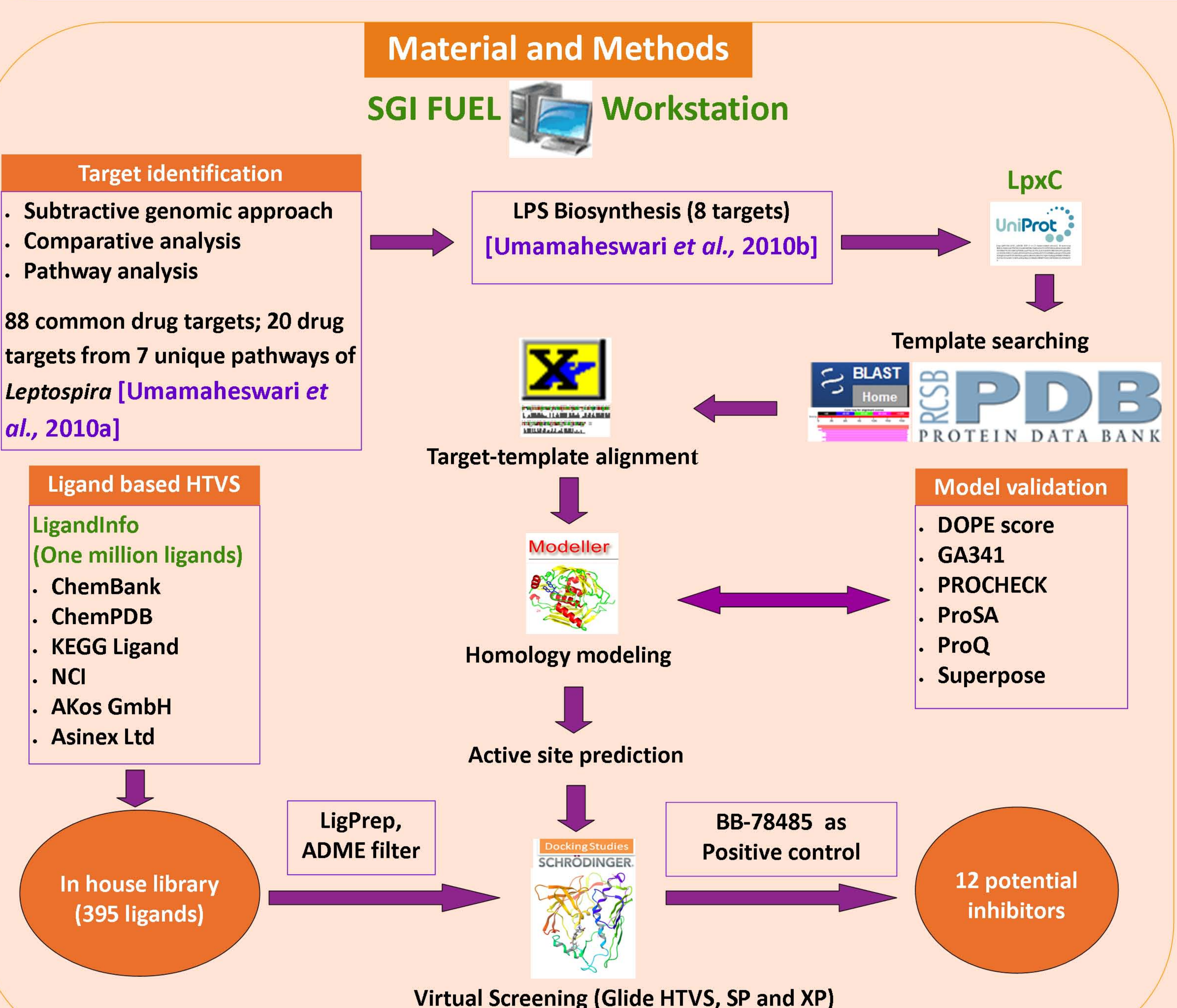

Virtual Screening (Glide HTVS, SP and XP)

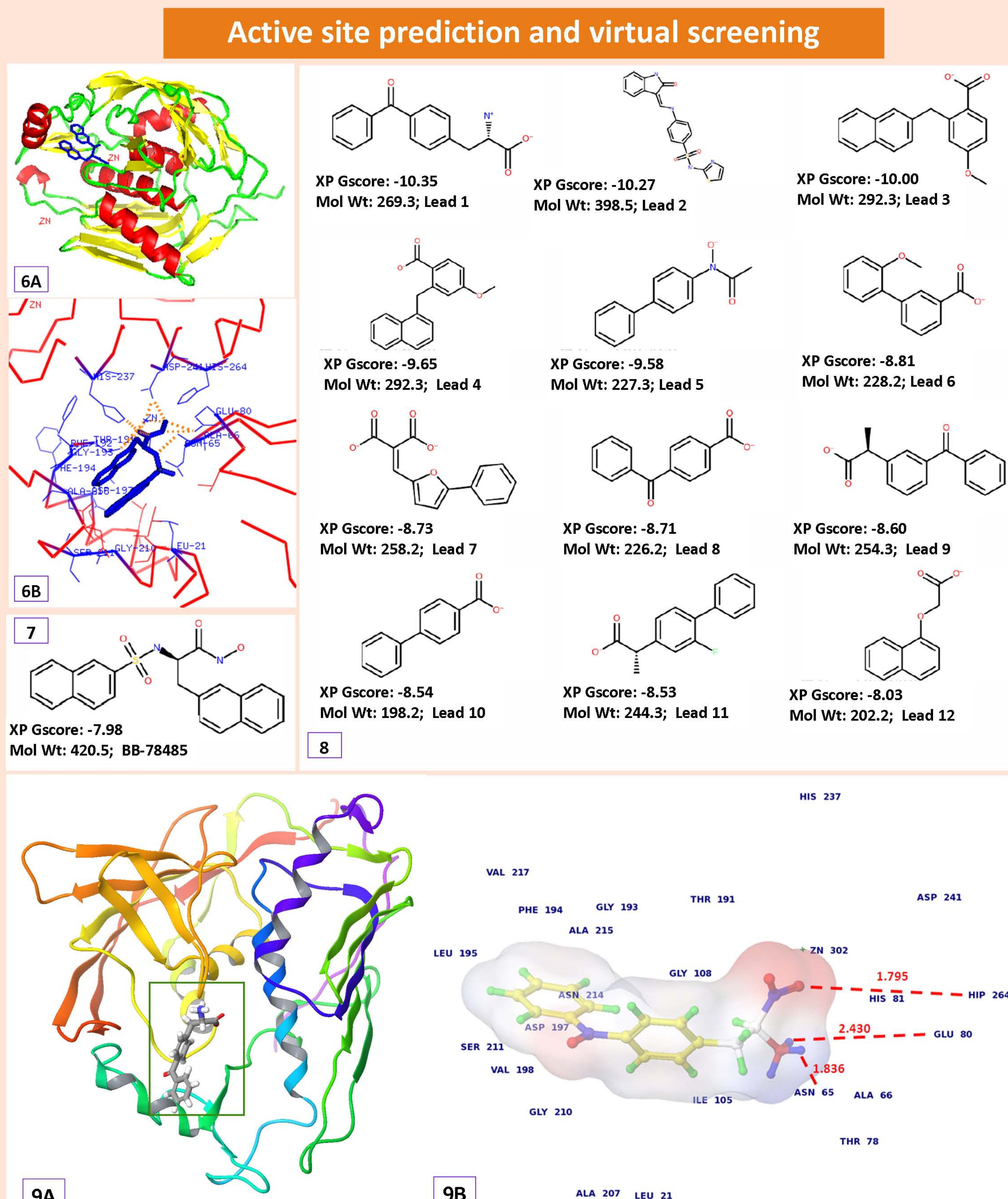

9A

$9 B$

Fig.6: (A) LpxC model in complex with BB-78485 (PMDB ID: PM0077228) (B) Predicted active site of IpxC.; Fig.7: BB-78485 with XPG score (Kcal/ mol) and molecular weight (Dalton); Fig.8: Proposed potential inhibitors of LpxC ; Fig.9: (A) Molecular docking complex of lpxC with paramol) and molecular weight (Dalton); $;$ Fg.8: Proposed potential inhibitors of LpxC; Fig.9: (A) Molecular docking complex of pides
(benzoyl)-phenylalanine (B) Docking of Para-(benzoyl)-phenylalanine showing hydrogen bonds and van der Waal interacting residues

\section{Conclusion}

Common drug targets from the unique pathways of pathogen are of significant interest towards designing drugs against leptospirosis. LPxC participates in unique LPS biosynthesis pathway and is common among all sequenced pathogenic Leptospira without any alternative mechanism to replace its catalytic activity. Thus, targeting IpxC for novel inhibitor discovery is worth mentioning.

The highly reliable IpxC 3D model with predicted active site residues paved the way for proposing 12 potential inhibitors and Para-(benzoyl)-phenylalanine as the best one to start with experimental validation towards designing anti-leptospirosis drug.

\section{Acknowledgements}

Authors are highly thankful to DBT, Ministry of Science and Technology, Govt. of India for supporting research at SVIMS Bioinformatics centre. We are also thankful to Dr. B. Vengamma, Director, SVIMS for her constant encouragement and support. 